
\title{
R Reserach S Suare \\ The Usage of High-Flow Nasal Oxygen (HFNO) for Patients with Covid-19 Outside Intensive Care Units
}

issa issa ( $\square$ issayacoubissa@gmail.com )

Sodersjukhuset AB

Marten Soderberg

Sodersjukhuset AB

Research

Keywords: High-flow nasal oxygen, Covid-19, ICU, ARDS

Posted Date: March 13th, 2021

DOI: https://doi.org/10.21203/rs.3.rs-285940/v1

License: (9) This work is licensed under a Creative Commons Attribution 4.0 International License.

Read Full License 


\section{Abstract}

\section{Introduction}

The usage of high-flow nasal oxygen (HFNO) has traditionally been located in intensive care units (ICU). As the Covid-19 pandemic continues to be a challenge to the whole world, health care providers are trying to manage space in the ICU. HFNO is well studied for usage in acute respiratory distress syndrome (ARDS). We tried to study the usage of HFNO in patients with moderate to severe ARDS related to Covid19 outside the ICU as well as its benefits both for patients and to offload the ICU.

\section{Methods}

We started a project to use HFNO in the ward of infectious diseases at Södersjukhuset, Stockholm. Patients with Covid-19 who needed HFNO were transferred to this ward after assessment from anesthesiologists or intensive care doctors. The patients were observed with frequent controls to assess the need of ICU in case of deterioration. The aim was mainly to offload the ICU.

\section{Results}

We studied 41 patients who were admitted for getting HFNO treatment either as primary treatment (StepUp) or after stabilizing in the ICU (Step-Down). We studied the patients' characteristics and the treatment's result and compared it to a subgroup of patients who did not survive this treatment. Most of our patients were men (80\%). They had at least one chronic disease (61\%). Each patient had on average 5.6 days of treatment with HFNO. $55 \%$ of the patients were discharged home or to geriatric rehabilitation. $10 \%$ avoided ICU completely and $15 \%$ of patients who simultaneously had a DNR orders (Do-Not-Resuscitate) survived by using HFNO and moved to geriatric rehabilitation. The usage of HFNO saved in total 229 days in the ICU. Mortality was higher among elderly patients, and patients with comorbidities (mainly hypertension and obesity).

\section{Discussion and conclusion}

HFNO treatment is feasible and efficient for patients with Covid-19, saving resources in ICU when the need is maximized, and offering additional advantages as waken proning and fewer complications compared to traditional ICU care. It requires however frequent controls as deterioration is recurrent.

\section{Introduction}

With millions of people affected, the novel Covid-19 pandemic is a serious challenge that has been among the most severe and widespread pandemics in modern history. About 3 billion people have needed to stay home in quarantine (1). A new challenge for health care providers is how to manage space in hospitals and increase the capacity of intensive care units to parallel the increasing need. Previous studies have shown that approximately $5 \%$ of the patients with Covid-19 have severe ARDS and are in need of intensive care (2-3). Sweden has fewer intensive care beds in comparison to other European 
countries(4). Therefore, it is a challenge to offer enough care to patients during the increasing need created by the pandemic.

Previous studies have shown that the usage of HFNO (high-flow nasal oxygen therapy) has helped patients with Covid-19-infection to both skip the ICU and receive better outcomes than ordinary oxygen treatment (5). HFNO is a technique whereby heated and humidified oxygen is delivered to the nose by using small nasal prongs, which patients describe as generally soft and pliable. When compared to traditional oxygen cannula or mask, HFNC (high-flow nasal cannula) has improved patient comfort in several studies (6-7-8). It would also avoid airway desiccation and epithelial injury at high flow rates.

This also generates low levels of positive pressure in the upper airways, permitting a higher fraction of minute ventilation to participate in alveolar gas exchange.

The high flow rates may also decrease physiological dead space by flushing expired carbon dioxide from the upper airway, a process that potentially explains the observed decrease in the work of breathing (68).

Several studies have shown that HFNO increases nasopharyngeal airway pressure that peaks at the end of expiration (6-7-8). This PEEP effect (Positive end-expiratory pressure) can potentially enhance oxygenation in patients with alveolar filling diseases such as acute respiratory distress syndrome (ARDS). The CPAP effect (continuous positive airway pressure) is greatest with the mouth closed, offering a mild to moderate effect. High flow rates have also been shown to result in an improved breathing pattern by increasing tidal volume and decreasing respiratory rate (9). At the same time the usage of HFNO under the pandemic of Covid-19 is considered to be aerosol-generating (which can mean more contagion) but there is a gap in evidence whether this translates into significant infection risk, how this risk compares with alternative respiratory supports and, perhaps most importantly, whether we can adequately protect ourselves from such aerosolization.

The aim of the study is to investigate the efficacy of HFNO in Covid-19 patients outside the ICU and its role in offloading the ICU.

\section{Methods}

At Södersjukhuset, Stockholm, a project to start HFNO treatment was initiated in the department of infectious diseases, with 15 single rooms with modern ventilation and negative suction pressure, built to be used for airborne diseases. This makes it possible to change the whole air in the room 8 times/hour, so the HFNO that is aerosol-generating would be safer for health workers. A negative pressure room is regarded as preferable for patients receiving HFNO according to the ANZICS guidelines (5). Initially, four rooms were supplied with HFNO and after four weeks the number increased to eight rooms. The number of rooms varied depending on the necessity, which was determined on a weekly basis, as well as the hospital's resources. Health care givers used maximized protection when patients had HFNO. 
An anesthesiologist or intensive care physician holds the decision-making about which patients are going to the infection ward to receive HFNO taking into consideration the ARDS severity that they have. This is evaluated with parameters including PFI ( $\mathrm{PaO} 2$ to $\mathrm{FiO} 2$ Index) which uses the partial pressure of the oxygen in blood ( $\mathrm{PaO} 2$ ) and the fraction of inspired oxygen ( $\mathrm{FiO} 2)$, and this comparison helps to determine the degree of any problems, such as ARDS, with how the lungs transfer oxygen to the blood (10). We used arterial blood gas analysis to measure PaO2 (Point-of-care testing). PFI was used to determine the need of mechanical respiration inclusive of HFNO. Severe ARDS is defined as a PFI of $<13$, while moderate PFI is defined as a PFI of $<26$. Normal PFI is $>50$.

The target for admission to infection ward was to offload the ICU. Patients who were stabilized with HFNO (irrespective of the previous need of invasive ventilation) in ICU; or needed HFNO under assessment of the necessity of intensive care were candidates for this treatment. Even patients who were unsuitable for tracheal intubation but were experiencing respiratory deterioration despite standard nasal oxygen or facemask oxygen were able to get HFNO treatment in the ward.

HFNO was initiated at $50 \mathrm{~L} / \mathrm{min}$ (AIRVO2 or Optiflow, Fisher \& Paykel Health Care Ltd., Auckland, New Zealand) with temperature set at $34 \mathrm{C}^{\circ}$. Nasal cannula size was determined by the patient's nostril size (50\%). FIO2 was adjusted to maintain SpO2 at 94\%. Flow and temperature were adjusted based on patient comfort and clinical response. These settings were accepted as standard settings except if the patient had other settings from the ICU when discharged to the ward. The settings ordered from the ICU were then retained.

In this retrospective, observational cohort study, we followed all patients with Covid-19 (defined with positive nasopharyngeal polymerase chain reaction for Severe Acute Respiratory Syndrome Corona Virus 2, SARS-CoV2-RNA PCR test) who were admitted to the infection ward to get HFNO between April and June 2020. We counted how many days the patients had HFNO treatment and how that saved valuable time in ICU. This was achieved by prolonging the time before initiating ICU care (which is called "step-up" and means primary usage of HFNO with frequent controls to evaluate the need of ICU and invasive ventilation), or by skipping it if the patient is stabilized with HFNO in the infection ward. Another possibility was to use the infection ward as a "step-down", which means helping patients who initially needed ICU care and then needed supportive respiratory care. Another patient category was patients with do-not-resuscitate and do-not-intubate orders where HFNO was considered as a viable treatment when other possibilities were limited as there was no intention to transfer to ICU despite deterioration.

The usage of HFNO as a step-up or step-down was assessed by looking at which ward the patient came from and was transferred to after the time in the infection ward. We even looked at the other diseases the patients had and studied other risk factors. The outcomes were the number of days of treatment with HFNO in the infection ward, the result of treatment (cure, death, or transfer to other hospital or care form), complications; and if patients had a do-not-resuscitate order (DNR) and how that affected the result. We compared the group as all with a subgroup of Covid-19 deaths who also had been treated with HFNO in the same ward. 
The project has been approved by the Swedish Ethical Review Authority (DNR 2020-03760).

\section{Statistical analysis}

Values are means (interquartile range) unless otherwise stated. P-values for differences obtained using Fisher's exact test and Kruskal Wallis test. Statistical significance was set at $p<0.05$. All statistical analyses were performed with the use of SPSS.

\section{Results}

Out of 41 patients treated with HFNO in the infection ward, $80 \%$ were men and $61 \%$ had at least one chronic disease. Patient demographics are described in Table 1. Patients were admitted within a median of 9.6 days (1-20) after the first SARS-CoV2 symptoms appeared. Each patient received HFNO treatment for 5.6 days on average, saving a total of 229 ICU-days.

Death occurred in 12 patients (29\%) where worsening conditions were noticed but there was no intention to transfer to ICU. It was considered as a preferable treatment for hypoxia rather than traditional oxygen masks. 6 patients (15\%) were transferred to the ICU due to deterioration.17 patients (41\%) could be discharged home after the treatment, and 6 patients $(15 \%)$ were discharged to geriatric rehabilitation. 4 patients (10\%) were admitted from the emergency department, 15 (39\%) from other departments; and HFNO was then used as a Step-Up in 20 cases. 21 patients (51\%) were admitted from the ICU units.

The usage of HFNO as Step-Down saved 126 ICU days. No intolerance of HFNO was noticed (It was already tested in the ICU). Mortality was considerably lower ( $2 / 21,9.5 \%$ vs $29 \%$ in the whole group). It helped 4 other patients (10\%) to avoid ICU completely. 2 other patients who received HFNO that simultaneously had a DNR order and no intention to transfer to ICU survived the infection and were moved to geriatric rehabilitation.

A significant difference was noticed in age between Covid-19 non-survivors and survivors (Mean 78 respectively 64 years old) ( $\mathrm{p} 0.001$ ). Comorbidities, mainly obesity and hypertension ( 0.03 respectively $0.05)$, were more frequent in patients who did not survive. No significant differences were noticed in other characteristics (sex, diabetes, heart failure, renal- and liver failure, COPD or smoking). No significant differences were noticed neither in CRP nor D-dimer. Patients who survived Covid-19 needed HFNO longer time (6 respectively $4, p$ 0.03). No differences were noticed in the frequency of pulmonary embolism or in the time having symptoms before visiting the hospital.

\section{Discussion}

SARS-COV-2 is an emerging contagious virus causing pneumonia, which sometimes needs hospitalization in the intensive care unit. As the cases increased, we tried to offload the ICU by using the infection ward for HFNO treatment. New studies began to show the importance and effectiveness of the use of HFNO not in ICU (11 12). 
The usage of HFNO helped us with valuable timesaving in the ICU, as we needed places in ICU for more critical cases, considering that the hospital had a total of 55 ICU spots when ICU capacity was fully utilized during the peak of the Covid-19 wave. At the same time, keeping frequent controls on patients with HFNO to transfer to ICU is of great importance (44\% deterioration).

To the best of our knowledge, this retrospective observational study for moderate and severe Covid-19 pneumonia is the largest reported to date in Europe, comparing with Covid-19 deaths and categorizing into usage as step-up and step-down. Our study showed that HFNO could successfully be used to provide respiratory support and avoid mechanical ventilation even in patients with profound hypoxemia. It resulted in offloading the ICU and was a considerable alternative for treatment of patients with DNR and DNI order.

One study in Cape Town, South Africa explored usage of HFNO in two resource-limited hospitals where HFNO was used primary in severe ARDS due to Covid-19 both in ICU and medical wards (11). About half of patients who got HFNO did not need ICU and it was successfully weaned, but nearly half the patients died. The patients got HFNO as a primary treatment (Step-up) and there were significant HIV and tuberculosis endemic components.

Covid-19 survivors and non-survivors in our study were admitted after approximately the same duration of symptoms, and no significant difference in CRP and Fibrin-D-dimer levels at baseline was noticed. Higher incidence of pulmonary embolism in the group of survivors can partly be explained by longer survival and thus more diagnostic procedures.

Our study did not compare patients receiving HFNO with those having COT (Conventional oxygen therapy). Based on other studies, there were no differences in mortality when using HFNO for acute hypoxemic respiratory failure (13). However, HFNO resulted in decreased requirement for tracheal intubation and had lower risk for escalation of oxygen therapy when compared with COT. It is important to note that the medical treatment was different during the times when Chloroquinephosphate was being used in the beginning to when Remdesivir was being recommended based on new studies. The usage of corticosteroids increased as more studies supported it. This was not included in the study. All the patients received anticoagulation either in full dose or for prophylaxis. Intermittent proning was used in most cases.

\section{Conclusion}

The usage of HFNO treatment would be useful outside the ICU most of all as a Step-Down as the patients are stabilized. The usage of HFNO as primary treatment can also be considered but requires frequent controls as the risk for deterioration is high. This study shows that HFNO treatment in non-critical care environment can offload the ICU units when the need for ICU and ventilators is maximized, and it is especially important as the threat of another Covid-19 wave approaches. 


\section{Declarations}

Ethics approval

The project has been approved by the Swedish Ethical Review Authority (DNR 2020-03760).

Consent for publication

Not applicable

Availability of data and materials

Not applicable

Competing interests

Not applicable

Funding

This research received no specific grant from any funding agency in the public, commercial, or not-forprofit sectors.

Authors' contributions

II conceived and drafted the article. MS recived the manuscript. Both authors read and approved the final manuscript.

Acknowledgements

Not applicable

\section{References}

- - Isaac Y, Prima A, Heidi L, et al. Social consequences of mass quarantine during epidemics: a systematic review with implications for the Covid-19 response. 2020 taaa192. doi:10.1093/jtm/taaa192https://pubmed.ncbi.nlm.nih.gov/33051660/.

- - Wei-jie G, Zheng-yi N, Yu H, et al. Clinical characteristics of coronavirus disease 2019 in China. N Engl J Med. 2020;382:1708-20. https://www.nejm.org/doi/full/10.1056/NEJMoa2002032 .

- - Halpern N, Pastores S. Critical care medicine beds, use, occupancy, and costs in the United States: A Methodological Review. Crit Care Med. 2015;43(11):2452-9.

http://europepmc.org/article/PMC/5520980.

- - Rhodes A, Ferdinande P, Flaatten H. The variability of critical care bed numbers in Europe. 
- Intensive C. Medicine 38,1647-1653(2012) https://link.springer.com/article/10.1007/s00134-0122627-8.

- - Lyons A, Callaghan M. The use of high-flow nasal oxygen in Covid-19. Anasthesia. 2020;75:843-7. https://doi.org/10.1111/anae.15073.

- - Tiruvoipati R, Lewis D, Haji K, et al. High-flow nasal oxygen vs high-flow face mask: a randomized crossover trial in extubated patients. J Crit Care. 2010;25:463.

https://pubmed.ncbi.nlm.nih.gov/19781896/.

- - Rittayamai N, Tscheikuna J, Rujiwit P. High-flow nasal cannula versus conventional oxygen therapy after endotracheal extubation: a randomized crossover physiologic study. Respir Care. 2014;59:485. https://pubmed.ncbi.nlm.nih.gov/24046462/.

- Williams R, Rankin N, Smith T, et al. Relationship between the humidity and temperature of inspired gas and the function of the airway mucosa. Crit Care Med 1996; 24:1920. https://pubmed.ncbi.nlm.nih.gov/8917046/.

- - Parke R, McGuinness S, Eccleston M. Nasal high-flow therapy delivers low level positive airway pressure. Br J Anaesth. 2009 Dec;103(6):886-90. https://pubmed.ncbi.nlm.nih.gov/19846404.

- - Acute Respiratory Distress Syndrome. The Berlin Definition. The ARDS Definition Task Force. J Am Med Ass. 2012;307(23):2526-33. doi:10.1001/jama.2012.5669. https://jamanetwork.com/journals/jama/article-abstract/1160659.

- - Calligaro G, Lalla U, Audley G. The utility of high-flow nasal oxygen for severe Covid-19 pneumonia in a resource-constrained setting: A multi-centre prospective observational study. 2020 Online first, 100570. https://www.thelancet.com/journals/eclinm/article/PIIS2589-5370(20)30314-X/fulltext.

- - Guy T, Créac'hcadec A, Ricordel C, et al. High-flow nasal oxygen: a safe, efficient treatment for Covid19 patients not in an ICU. European Respiratory Journal. Jan 2020, 2001154; DOI:

10.1183/13993003.01154-2020. https://erj.ersjournals.com/content/early/2020/08/03/13993003.01154-2020.

- - Rochwerg B, Granton D, Wang DX, et al. High flow nasal cannula compared with conventional oxygen therapy for acute hypoxemic respiratory failure: a systematic review and meta-analysis. Intensive Care Med. 2019;45:563-72. https://pubmed.ncbi.nlm.nih.gov/30888444/.

\section{Table}

Table 1: characteristics of patients with Covid-19 with HFNO treatment in the infection ward 


\begin{tabular}{|c|c|c|c|c|}
\hline eter & $\begin{array}{l}\text { All patients } \\
(\mathrm{n}=41)\end{array}$ & $\begin{array}{l}\text { Non-survivors } \\
(\mathrm{n}=12)\end{array}$ & $\begin{array}{l}\text { Survivors } \\
(\mathrm{n}=29)\end{array}$ & $\begin{array}{l}\mathrm{p} \text { - } \\
\text { value }\end{array}$ \\
\hline sex, No (\%) & $33(80)$ & $10(85)$ & $23(80)$ & 0.7 \\
\hline ears (range) & $68(48-88)$ & $78(72-88)$ & $64(48-88)$ & 0.001 \\
\hline$: 30 \mathrm{~kg} / \mathrm{m}^{2}$, No (\%) & $4(10)$ & 3 & 1 & 0.03 \\
\hline co use, No (\%) & $6(12)$ & 3 & 3 & 0.2 \\
\hline tension, No (\%) & $18(44)$ & 8 & 10 & 0.05 \\
\hline tes, No (\%) & $9(22)$ & 3 & 6 & 0.7 \\
\hline or liver failure, & $6(15)$ & 2 & 4 & 0.8 \\
\hline failure, No (\%) & $7(17)$ & 4 & 3 & 0.08 \\
\hline , No (\%) & $5(12)$ & 3 & 2 & 0.1 \\
\hline Irder, No (\%) & $14(34)$ & 12 & 2 & $<0.001$ \\
\hline rder, No (\%) & $14(34)$ & 12 & 2 & $<0.001$ \\
\hline mg/L (range) & $150(6-500)$ & 112 & 165 & 0.15 \\
\hline $\begin{array}{l}\text {-D-dimer } *, \mathrm{mg} / \mathrm{L} \\
\text { ?) }\end{array}$ & $3.7(0,25-35)$ & 1.8 & 4.4 & 0.3 \\
\hline on of symptoms, & $9.6(1-20)$ & 9.0 & 9.8 & 0.49 \\
\hline Idex (range) & $14(7,5-29)$ & 14 & 14 & 0.9 \\
\hline nary embolism, & $8(20)$ & 1 & 7 & 0.2 \\
\hline rance for HFNC, & $2(5)$ & 1 & 1 & 0.5 \\
\hline $\begin{array}{l}\text { ising HFNO, } \\
\text { ) }\end{array}$ & $5.6(1-14)$ & 3.9 & 6.3 & 0.03 \\
\hline
\end{tabular}

Characteristics of 41 patients with Covid-19 and HFNO treatment admitted to the infection ward at Södersjukhuset, Stockholm, between April and June 2020.

Values are means (\%) unless otherwise stated.

DNR, Do-not-resuscitate; DNI, Do-not-intubate; COPD, Chronic obstructive pulmonary disease; CRP, Creactive protein; PFI, $\mathrm{PaO} 2 / \mathrm{FiO} 2$ index.

*When the treatment was initiated 